\title{
Desarrollo de un atlas fotográfico de porciones de alimentos venezolanos
}

\author{
Pablo Hernández ${ }^{a, *}$, Jennifer Bernal ${ }^{b}$, Mirla Morón $^{a}$, Yuly Velazco $^{a}$, Elsy Oráa ${ }^{a}$, Claret Mata ${ }^{b}$ \\ a Universidad Central de Venezuela, Escuela de Nutrición y Dietética, Venezuela. \\ b Universidad Simón Bolívar, Departamento de Tecnología de Procesos Biológicos y Bioquímicos, Venezuela. \\ *doctuscumliber@gmail.com
}

Recibido el 31 de octubre de 2014; aceptado el 13 de enero de 2015.

Desarrollo de un atlas fotográfico de porciones de alimentos venezolanos

\section{PALABRAS CLAVE}

Desarrollo de instrumento;

Fotografía;

Atlas;

Tamaño de la porción;

Encuestas nutricionales;

Evaluación dietética;

Venezuela.

\section{RESUMEN}

Introducción: Las herramientas de ayuda para estimar las porciones de alimento ingeridas representan un factor clave para elevar la calidad de los datos obtenidos en las encuestas de consumo alimentario. Hasta el momento, Venezuela no cuenta con alguna herramienta, debidamente validada y publicada, por tal motivo, el objetivo de este estudio fue desarrollar un atlas fotográfico como herramienta complementaria para evaluar, en adultos venezolanos, el consumo habitual de alimentos.

Material y Métodos: Se establecieron 13 grupos de alimentos de acuerdo a su contenido de nutrientes, según la Tabla de Composición de Alimentos de Venezuela y la Hoja de Balance de Alimentos venezolana. Se seleccionaron los de consumo más frecuente por los venezolanos según datos de investigaciones nacionales y otros estudios adelantados por los autores. Para cada alimento se establecieron 3 tamaños de porciones: pequeña, mediana y grande; las cuales se pesaron mediante una balanza electrónica antes de proceder a fotografiarlas. Finalmente se procedió a un proceso de valoración por expertos.

Resultados: El instrumento propuesto incluye 110 alimentos con un total de 300 fotografías, distribuidas en 100 láminas, producto de cuidadosas fases de planificación y diseño de instrumentos, incluyendo la revisión de los materiales de referencia nacional e internacional y contando con la evaluación satisfactoria de la pertinencia de los ítems y la relevancia de la información del atlas, por parte de los expertos consultados.

Conclusiones: El atlas fotográfico de alimentos desarrollado, según la opinión de los expertos revisores, podría resultar una herramienta útil para ser usada en la evaluación del consumo alimentario en adultos venezolanos. No obstante, para cumplir con dicho propósito, el instrumento propuesto debería ser validado para comprobar sus condiciones técnicas de validez y confiabilidad. 
Development of a photographic atlas of Venezuelan food portions

\section{KEYWORDS}

Instrument development;

Photography;

Atlases;

Portion size;

Nutritional surveys;

Dietary assessment;

Venezuela.

\section{ABSTRACT}

Introduction: The instruments to estimate consumed portion are a key factor, which ensures data quality in surveys of food intake assessment. Venezuela does not have a tool like this, properly validated and published. The aim of the study was develop a photographic atlas as a complementary tool for assess usual food consumption in Venezuelan adults.

Material and Methods: Foods were classified into 13 groups according to their similarity in nutrient content according to the table of composition and the food balance sheet of Venezuela. We selected the most consumed by Venezuelans according to data from national studies and another researches by the authors. For each food were set 3 portion sizes: small, medium and large; which were weighed in an electronic balance before taking a picture. Later, the atlas underwent a valuation process by experts.

Results: The instrument includes 110 foods with a total of 300 photographs, distributed in 100 sheets, presenting the advantages of quality control used during the planning and design, the review of national and international reference materials. The experts successfully assessed the pertinence of the items and the relevance of the atlas information.

Conclusions: The photographic atlas of foods developed, in the opinion of the expert reviewers, could be a useful tool to be used in the evaluation of food consumption in Venezuelan adults. However, to fulfill this purpose, the proposed instrument should be validated to prove their technical conditions of validity and reliability.

\section{CITA}

Hernandez P, Bernal J, Morón M, Velazco Y, Oráa E, Mata C. Desarrollo de un atlas fotográfico de porciones de alimentos venezolanos. Rev Esp Nutr Hum Diet. 2015; 19(2): 68 - 76. DOI: 10.14306/renhyd.0.0.132

\section{INTRODUCCIÓN}

Uno de los procesos clave en la realización de las encuestas de consumo de alimentos es la estimación precisa del tamaño de las porciones consumidas, pues algún error en esta fase trae como consecuencia un sesgo en el cálculo de los aportes de energía y nutrientes de las comidas, de allí la importancia de lograr la mayor precisión en este paso ${ }^{1}$.

Son múltiples las alternativas utilizadas como ayudas visuales para reducir el error en la estimación del consumo alimentario, entre las que pueden nombrarse los modelos de alimentos ${ }^{2}$, utensilios de cocina (platos, vasos), medidas prácticas (tazas y cucharadas) ${ }^{3}$ y plantillas rectangulares o circulares bidimensionales de distintos tamaños ${ }^{4}$.

Los atlas de fotografías de alimentos constituyen otros instrumentos útiles para recolectar los datos de las encuestas de consumo de alimentos, puesto que ayudan a la estima- ción y descripción de las raciones ingeridas. Su uso permite que el entrevistado identifique una imagen determinada para indicar los gramos o mililitros consumidos 5 . Sin embargo, estas colecciones fotográficas no son de uso frecuente en la mayoría de los estudios que evalúan el consumo de alimentos, quizás porque deben ser sometidas a un proceso de validación para asegurar su confiabilidad, además, de existir escasa información al respecto a nivel latinoamericano.

Algunas experiencias anteriores en las cuales evaluaron la concordancia entre la porción servida y la ración estimada, demostraron un grado de correlación entre el 50 y el $70 \%$, además de que su uso mejora la estimación de las porciones consumidas cuando reemplaza la idea de porción promedio o mediana ${ }^{1,6}$.

Hasta el presente no se ha realizado en Venezuela un atlas fotográfico de alimentos debidamente validado y publicado. Por este motivo, el objetivo del presente estudio 
fue desarrollar un atlas fotográfico como herramienta complementaria para evaluar el consumo habitual de alimentos en adultos venezolanos. Los resultados que se presentan corresponden a la primera fase de diseño de instrumentos, puesto que no se incluyen pruebas de validez y confiabilidad.

\section{MATERIAL Y MÉTODOS}

Como inicio para el desarrollo del atlas fotográfico propuesto, se realizó una revisión bibliográfica en las bases de datos de Scielo Venezuela y Google Scholar en busca de atlas fotográficos de alimentos que hayan sido realizados y validados en el país. Las palabras clave de búsqueda fueron: atlas de alimentos, consumo de alimentos, atlas fotográfico de alimentos y Venezuela.

\section{Grupos y lista de alimentos}

En primer lugar se elaboró una clasificación por grupos de alimentos con similar contenido de nutrientes, en las mismas categorías establecidas por la Tabla de Composición de Alimentos ${ }^{7}$ y la Hoja de Balance de Alimentos venezolana ${ }^{8}$.

Para seleccionar los alimentos a ser incluidos en el atlas se usaron los datos presentados en la Encuesta de Seguimiento al Consumo de Alimentos (segundo semestre 2010 al segundo semestre de 2012) ${ }^{9}$ y la Encuesta Nacional de Consumo de Alimentos ${ }^{10}$, estudios nacionales del Instituto Nacional de Estadística (INE) así como el estudio de Hábitos Alimenticios $^{11}$ del Banco Central de Venezuela. Además se realizó una prueba para verificar la presencia de los alimentos más representativos de cada grupo seleccionado a través de un trabajo piloto con 50 pacientes venezolanos, cuyas edades oscilaban entre los 18 y 61 años de edad captados en el servicio de endocrinología del Hospital Militar de Caracas "Dr. Carlos Arvelo", a los cuales se les aplicó un recordatorio del consumo de alimentos en un día usual o típico ${ }^{3}$.

\section{Tamaño de las porciones}

Se establecieron 3 opciones de tamaño de las porciones para cada alimento en orden ascendente: pequeña, mediana y grande. Sin embargo, se puede elegir una porción intermedia entre las raciones pequeña y mediana o mediana y grande, la cual puede ser cuantificada igualmente a través del instrumento. Las opciones de raciones de alimentos se elaboraron tomando como referencia las cantidades repor- tadas en el estudio previo ${ }^{3}$ con los 50 sujetos mencionados, así como las Tablas de Raciones de Alimentos - UCV ${ }^{12}$. Cabe mencionar que esta última, es una herramienta obtenida como producto de una investigación institucional y que a pesar de ser de uso común, no ha sido validada ni publicada.

\section{Las fotografías de los alimentos}

Se tomaron fotografías a color, colocando las raciones de alimentos en un plato blanco estándar de $25 \mathrm{~cm}$ o un vaso transparente de 355 cc de capacidad, contrastado con un fondo azul. Cada una de las fotografías incluidas en el atlas, fueron tomadas a alimentos reales, especialmente preparados para realizar las fotografías, no se utilizó la adición de colorantes o técnicas de mejoramiento de imagen, ni fue utilizado ningún programa informático de edición de fotografías. Esto debido a que la utilidad del atlas es para estimar la ración consumida, sin pretender expresar la ración que se desea consumir.

Para cada alimento se pesó la ración a ser fotografiada haciendo uso de una balanza electrónica con $1 \mathrm{~g}$ de precisión (TANITA ${ }^{\circledR}$ KD-310SV). Cada foto se tomó con un ángulo aproximado de $90^{\circ}$ y con una distancia de $50 \mathrm{~cm}$, siguiendo el criterio de Lazarte ${ }^{13}$ en la elaboración de un atlas fotográfico de alimentos para Bolivia. Se hizo uso de una iluminación fluorescente de tipo blanco cálido con una potencia de 500W con una temperatura de color de 3200K y una cámara digital (Kodak ${ }^{\circledR}$ C530) con un modo de disparo automático. Las imágenes se establecieron con un tamaño similar en cada lámina de 7,5 x 7,5 cms.

Con el fin de facilitar la toma fotográfica y simplificar el atlas desarrollado, se contempló que en algunos casos, una sola lámina pudiera contener dos o más ítems de alimentos cuya imagen fotográfica sea similar como por ejemplo la leche completa, semidescremada y descremada.

\section{Valoración por expertos}

Obtenida la primera versión del cuestionario se pasó a un proceso de revisión y valoración por expertos, contando con la participación voluntaria de 3 profesionales de reconocida trayectoria y experiencia de trabajo en el área. Se utilizó una pauta en la que se pidió a los expertos su valoración acerca de la pertinencia de los ítems de alimentos y la relevancia de la información que puede aportar el atlas propuesto. Por otro lado, se les pidió su opinión acerca de la adecuación de las distintas fuentes de recolección de información y, por último, la pertinencia del objetivo de la investigación. 


\section{RESULTADOS}

El proceso de búsqueda electrónica arrojó 9 referencias, provenientes de la búsqueda en Google Scholar, debido a que en la base de datos de Scielo Venezuela no se encontró alguna referencia. De las 9 referencias, 3 de ellas fueron libros sobre nutrición, 2 citas de publicaciones científicas, 2 trabajos de grado y 2 artículos científicos. Tras su revisión a totalidad se encontró que hasta el momento no existe algún atlas fotográfico de alimentos similar válido y de libre acceso.

La lista preliminar de alimentos frecuentemente consumidos se conformó con 163 ítems provenientes de las fuentes consultadas ${ }^{9,10,11}$; posteriormente tras la verificación con el estudio piloto ${ }^{3}$, se seleccionaron 107 alimentos que en su mayoría (92\%) fueron consumidos por más de 40 pacientes $(>80 \%)$ y presentaban un contenido nutricional de interés. Además, el grupo de expertos consultado recomendó la inclusión de 3 alimentos.

La versión final del atlas fotográfico incluye 110 alimentos diferentes, distribuidos en los 13 grupos de alimentos seleccionados, de la siguiente manera: 1.- Cereales y derivados (13 alimentos); 2.- Raíces, tubérculos y otros feculentos (9 alimentos); 3.- Leguminosas (4 alimentos); 4.Azúcar, miel y edulcorantes (4 alimentos); 5.- Hortalizas (17 alimentos); 6.- Frutas (13 alimentos); 7.- Leche y derivados (7 alimentos); 8.- Huevos (3 alimentos); 9.- Carnes (7 alimentos); 10.- Pescados y mariscos (6 alimentos); 11.Aceites, grasas y semillas oleaginosas (7 alimentos); 12.Bebidas alcohólicas y no alcohólicas (11 alimentos); 13.Misceláneos (9 alimentos).

En 8 oportunidades se agruparon en una sola lámina algunos alimentos con similar imagen fotográfica, tamaño de la porción y volumen. Estos fueron: azúcar blanca, azúcar morena y fructosa; leche completa, semidescremada y descremada; yogurt completo y semidescremado; cerveza regular y light; jugo pasteurizado regular y light; bebidas gaseosas regulares y light; chocolate regular y light; gelatina regular y light. Posterior a esta agrupación, los 110 alimentos seleccionados se distribuyeron en 100 láminas con 3 fotografías cada una; de las cuales, una corresponde a la ración pequeña, otra a la mediana y una a la grande, tal como lo establece el método de elaboración de un atlas fotográfico ${ }^{15}$. La lista de láminas de alimentos y las raciones pequeñas, medianas y grandes pueden observarse en la Tabla 1.

Tabla 1. . Lista de láminas de alimentos seleccionados con el tamaño de la ración pequeña, mediana y grande.

\begin{tabular}{l:l} 
N & Alimento \\
\hdashline 1 & Pan blanco \\
3 & Pan integral \\
4 & Arepa (torta de pan de maíz) \\
5 & Arroz blanco \\
6 & Arroz integral \\
7 & Pasta blanca \\
8 & Pasta integral \\
9 & Cereal de desayuno \\
10 & Cereal integral \\
11 & Avena en hojuelas (en escamas) \\
12 & Galletas saladas \\
13 & Galletas dulces \\
14 & Galletas integrales \\
15 & Papa cocida con concha (patata cocida con piel) \\
16 & Papa cocida sin concha (patata cocida sin piel) \\
17 & Papas fritas (patatas fritas)
\end{tabular}

\begin{tabular}{|c|c|}
\hline Ración Pequeña & Ración Mediana \\
\hline 1 Reb. / 24g & 2 Reb. / 48g \\
\hline 1 Reb. / 28g & 2 Reb. / 56g \\
\hline 1 Ud. / 50g & 1 Ud. / 150g \\
\hline $1 / 2 \mathrm{Tz} . / 80 \mathrm{~g}$ & $1 \mathrm{Tz} . / 160 \mathrm{~g}$ \\
\hline $1 / 2$ Tz. / 80g & $1 \mathrm{Tz} . / 160 \mathrm{~g}$ \\
\hline $1 / 2$ Tz. / 75g & $1 \mathrm{Tz} . / 150 \mathrm{~g}$ \\
\hline $1 / 2$ Tz. / 75g & $1 \mathrm{Tz} . / 150 \mathrm{~g}$ \\
\hline $1 / 2 \mathrm{Tz} . / 18 \mathrm{~g}$ & $1 \mathrm{Tz} . / 36 \mathrm{~g}$ \\
\hline $1 / 2$ Tz. / $28 \mathrm{~g}$ & $1 \mathrm{Tz} . / 56 \mathrm{~g}$ \\
\hline $1 \mathrm{Cda} . / 5 \mathrm{~g}$ & 3 Cdas. / 15g \\
\hline 1 Env. / 24g & 2 Env. / 48g \\
\hline 1 Env. / 28g & 2 Env. / 56g \\
\hline 1 Env. / 28g & 2 Env. / 56g \\
\hline $1 / 2$ Ud. / 90g & 1 Ud. / 180g \\
\hline $1 / 2$ Ud. / 75g & 1 Ud. / 150g \\
\hline $1 / 2$ Rac. / 60g & 1 Rac. / 120g \\
\hline 1 Troz. / 75g & 2 Troz. / 150g \\
\hline
\end{tabular}

Ración Grande 4 Reb. / $96 \mathrm{~g}$

4 Reb. / 112g

1 Ud. / 250g

2 Tz. / 320g

2 Tz. / 320g

2 Tz. / 300g

2 Tz. / 300g

2 Tz. / $72 \mathrm{~g}$

2 Tz. / $112 \mathrm{~g}$

6 Cdas. / 30g

4 Env. / 96g

4 Env. / 112g

4 Env. / 112g

3 Uds. / 540g

3 Uds. / 450g

2 Rac. / 240g

4 Troz. / 300g 
Apio, Ocumo, Ñame

Plátano verde sancochado (hervido)

Plátano verde frito (tostón)

Plátano maduro sancochado (hervido)

Plátano maduro frito (tajadas)

Caraotas negras (tipo de fríjol o alubia)

Lentejas

Arvejas (guisante)

Otros Granos

Azúcar blanca, morena / Fructosa

Miel de abeja

Edulcorantes

Vegetales (aliños)

Cebolla

Lechuga

Tomate perita

Calabacín

Zanahoria

Berenjena

Pepino

Auyama (calabaza)

Berro

Brócoli

Espinaca

Remolacha

Repollo

Vainita (judía verde o habichuela)

Coliflor

Maíz dulce

Lechosa (papaya)

Cambur (banana)

Melón

Guayaba

Naranja

Patilla (sandía)

Pera

Manzana

Durazno (melocotón)

Piña

Mango

Parchita (maracuyá)

Uva

Leche completa, semi o descremada

Yogurt entero o descremado
$1 / 2$ Tz. / $75 \mathrm{~g}$

$1 / 4$ Ud. / 60g

$1 / 4$ Ud. / $25 g$

$1 / 4$ Ud. / $55 \mathrm{~g}$

$1 / 4$ Ud. / 20g

$1 / 4$ Tz. / 58g

$1 / 4$ Tz. / 57g

$1 / 4$ Tz. / 59g

$1 / 4$ Tz. / 40g

1 Cdta. / 5g

1 Cdta. / 5g

1 Sobre / $1 \mathrm{~g}$

1 Cda. / $5 \mathrm{~g}$

$1 \mathrm{Cda} / \mathrm{10g}$

$1 / 2$ Ноја / 11g

$1 / 2$ Tz. / 40g

$1 / 4$ Tz. / $25 \mathrm{~g}$

$1 / 4$ Tz. / $20 \mathrm{~g}$

$1 / 2$ Tz. / $50 \mathrm{~g}$

$1 / 4$ Tz. / 30g

$1 / 2$ Tz. / $68 \mathrm{~g}$

$1 / 2$ Tz. / $25 \mathrm{~g}$

$1 / 2$ Tz. / 48g

$1 / 4$ Tz. / $33 \mathrm{~g}$

$1 / 4$ Tz. / 40g

$1 / 2$ Tz. / 38g

$1 / 4$ Tz. / 33g

$1 / 2$ Tz. / 65g

$1 \mathrm{Cda}$ / $10 \mathrm{~g}$

$1 / 2$ Tz. / $80 \mathrm{~g}$

$1 / 2$ Ud. / 50g

$1 / 2$ Tz. / $80 \mathrm{~g}$

$1 / 2$ Ud. / $71 \mathrm{~g}$

$1 / 2$ Ud. / 60g

$1 / 2$ Tz. / 100g

$1 / 2$ Ud. / 115g

$1 / 2$ Ud. / 110g

1 Ud. / $76 \mathrm{~g}$

1 Rued. / 75g

1 Ud. / 240g

$1 / 2$ Ud. / 50g

5 Uds. / 60g

$1 / 2$ Vaso / 120cc

$1 / 2$ Vaso / $120 c c$
$1 \mathrm{Tz} . / 150 \mathrm{~g}$

$1 / 2$ Ud. / 120g

$1 / 2$ Ud. / $50 \mathrm{~g}$

$1 / 2$ Ud. / 110g

$1 / 2$ Ud. / 60g

$1 / 2$ Tz. / 116g

$1 / 2$ Tz. / $114 \mathrm{~g}$

$1 / 2$ Tz. / $118 \mathrm{~g}$

$1 / 2$ Tz. / $80 \mathrm{~g}$

$1 \mathrm{Cda}$ / 10g

$1 \mathrm{Cda} / \mathrm{10g}$

2 Sobres / $2 g$

$1 \mathrm{Cda} / \mathrm{10g}$

$1 \frac{1}{2}$ Cdas. / $15 \mathrm{~g}$

1 Hoja / 22g

1 Tz. / 80g

$1 / 2$ Tz. / $50 \mathrm{~g}$

$1 / 2$ Tz. / $40 \mathrm{~g}$

$1 \mathrm{Tz} . / 100 \mathrm{~g}$

$1 / 2$ Tz. / 60g

$1 \mathrm{Tz} . / 135 \mathrm{~g}$

$1 \mathrm{Tz}$ / 50g

1 Tz. / 95g

$1 \mathrm{Tz} . / 130 \mathrm{~g}$

$1 \mathrm{Tz} . / 160 \mathrm{~g}$

$1 \mathrm{Tz} . / 75 \mathrm{~g}$

$1 / 2$ Tz. / $65 \mathrm{~g}$

$1 \mathrm{Tz} . / 130 \mathrm{~g}$

3 Cdas. / 30g

$1 \mathrm{Tz}$ / / 160g

1 Ud. / 100g

$1 \mathrm{Tz}$ / $160 \mathrm{~g}$

1 Ud. / 142g

1 Ud. / 120g

$1 \mathrm{Tz}$ / / 200g

1 Ud. / 230g

1 Ud. / 220g

2 Uds. / 152g

2 Rued. / 150g

2 Uds. / 480g

1 Ud. / 100g

8 Uds. / 98g

1 Vaso / 240cc

1 Vaso / 240cc
2 Tz. / 300g

1 Ud. / 240g

1 Ud. / 150g

1 Ud. / 220g

1 Ud. / 100g

1 Tz. / 232g

1 Tz. / 228g

1 Tz. / 236g

$1 \mathrm{Tz} . / 160 \mathrm{~g}$

2 Cdas. / 20g

2 Cdas. / $20 \mathrm{~g}$

4 Sobres / $4 \mathrm{~g}$

2 Cdas. / 20g

2 Cdas. / 20g

3 Hojas / 66g

2 Tz. / 160g

$1 \mathrm{Tz} . / 100 \mathrm{~g}$

$1 \mathrm{Tz} . / 80 \mathrm{~g}$

2 Tz. / 200g

$1 \mathrm{Tz} . / 120 \mathrm{~g}$

2 Tz. / 270g

2 Tz. / 100g

2 Tz. / 190g

2 Tz. / $260 \mathrm{~g}$

2 Tz. / 320g

2 Tz. / 150g

$1 \mathrm{Tz} . / 130 \mathrm{~g}$

$11 / 2$ Tz. / 195g

5 Cdas. / 50g

3 Tz. / 480g

3 Ud. / 300g

3 Tz. / 480g

3 Uds. / 426g

3 Uds. / 360g

3 Tz. / 600g

2 Uds. / 460g

2 Uds. / 440g

3 Uds. / 228g

4 Rued. / 300g

3 Uds. / 720g

3 Uds. / 300g

16 Uds. / 200g

3 Vasos / 720cc

3 Vasos / 720cc 


\begin{tabular}{|c|c|c|c|c|}
\hline 62 & Quesos blancos & 1 Reb. / 25g & 3 Reb. / 75g & 6 Reb. / 150g \\
\hline 63 & Quesos amarillos & 1 Reb. / 25g & 3 Reb. / 75g & 6 Reb. / 150g \\
\hline 64 & Huevo entero de gallina & 1 Ud. / 53g & 2 Uds. / 106g & 4 Uds. / 212g \\
\hline 65 & Huevo frito & $1 \mathrm{Ud} . / 60 \mathrm{~g}$ & 2 Uds. / 120g & 4 Uds. / 240g \\
\hline 66 & Claras de huevo & 1 Ud. / 30g & 2 Uds. / 60g & 4 Uds. / 120g \\
\hline 67 & Carne de pollo con piel & 1 Pza. / 71g & 2 Pzas. / 144g & 3 Pzas. / 257g \\
\hline 68 & Carne de pollo sin piel & $1 / 2$ Filet. / $75 \mathrm{~g}$ & 1 Filet. / 150g & 2 Filet. / 300g \\
\hline 69 & Pechuga de pollo & 1 Reb. / 20g & 3 Reb. / 60g & 6 Reb. / 120g \\
\hline 70 & Carne de res & 1/2 Bistec / 50g & 1 Bistec / 100g & 3 Bistecs / 300g \\
\hline 71 & Carne de cerdo & 1/2 Pza. / 46g & 1 Pza. / 92g & 3 Pzas. / 276g \\
\hline 72 & Jamón de cerdo & 1 Reb. / 20g & 3 Reb. / 60g & 6 Reb. / 120g \\
\hline 73 & Pechuga de pavo & 1 Reb. / 20g & 3 Reb. / 60g & 6 Reb. / 120g \\
\hline 74 & Pescado blanco no frito & 1 Filet. / 75g & 2 Filet. / 150g & 3 Filet. / 225g \\
\hline 75 & Pescado blanco frito & 1 Filet. / 90g & 2 Filet. / 180g & 3 Filet. / 270g \\
\hline 76 & Pescado azul no frito & 1 Filet. / 40g & 2 Filet. / 80g & 3 Filet. / 1200g \\
\hline 77 & Pescado azul frito & 1 Filet. / 43g & 2 Filet. / 86g & 3 Filet. / 129g \\
\hline 78 & Pescado azul enlatado & $1 / 4 \mathrm{Tz} . / 45 \mathrm{~g}$ & 1/2 Tz. / 90g & $11 / 2$ Tz. / 270g \\
\hline 79 & Mariscos & 1 Rac. / 33g & 2 Rac. / 66g & 3 Rac. / 198g \\
\hline 80 & Aceite vegetal & 1 Cdta. / 5g & 1 Cda. / 10g & 2 Cdas. / 20g \\
\hline 81 & Margarina & 1 Cdta. / 5g & $1 \mathrm{Cda} . / 10 \mathrm{~g}$ & 2 Cdas. / 20g \\
\hline 82 & Mayonesa & 1 Cdta. / 5g & $1 \mathrm{Cda} . / 10 \mathrm{~g}$ & 2 Cdas. / 20g \\
\hline 83 & Maní (cacahuete) & $1 / 2$ Rac. / 15g & 1 Rac. / 30g & 3 Rac. / 90g \\
\hline 84 & Otros frutos secos & $1 / 2 \operatorname{Rac} . / 15 g$ & 1 Rac. / 30g & 3 Rac. / 90g \\
\hline 85 & Aguacate & $1 / 8$ Ud. / 60g & $1 / 4$ Ud. / 120g & $1 / 2$ Ud. / 240g \\
\hline 86 & Vino & $1 / 2$ Copa / 60cc & 1 Copa / 120cc & 2 Copas /240cc \\
\hline 87 & Cerveza y light & $1 / 2$ Beb. / 178cc & 1 Beb. / 355cc & 3 Beb. / 1065cc \\
\hline 88 & Bebidas espirituosas & 1/2 Porción / 25cc & 1 Porción / 50cc & 3 Porciones / 150cc \\
\hline 89 & Café infusión & $1 / 4$ Vaso / 60cc & 1 Vaso / 240cc & 2 Vasos / 480cc \\
\hline 90 & Té infusión & $1 / 4$ Vaso / 60cc & 1 Vaso / 240cc & 2 Vasos / 480cc \\
\hline 91 & Naranjada & $1 / 2$ Vaso / 120cc & 1 Vaso / 240cc & 3 Vasos / 720cc \\
\hline 92 & Jugo pasteurizado y light & $1 / 2$ Vaso / 120cc & 1 Vaso / 240cc & 3 Vasos / 720cc \\
\hline 93 & Bebidas gaseosas & $1 / 2$ Vaso / 120cc & 1 Vaso / 240cc & 3 Vasos / 720cc \\
\hline 94 & Helados de crema & $1 / 2$ Rac. / 25g & 1 Rac. / 50g & 3 Rac. / 150g \\
\hline 95 & Chocolate y light & $1 / 2$ Rac. / 15g & 1 Rac. / 30g & 3 Rac. / 90g \\
\hline 96 & Caramelos & 2 Uds. / 10g & 5 Uds. / 25g & 10 Uds. / 50g \\
\hline 97 & Gelatina & 1 Copa / 100g & 2 Copas / 200g & 3 Copas / 300g \\
\hline 98 & Ketchup & 1 Cdta. / 5g & $1 \mathrm{Cda} . / 10 \mathrm{~g}$ & 2 Cdas. / 20g \\
\hline 99 & Pizza & 1 Porción / 66g & 2 Porciones / 132g & 4 Porciones / 264g \\
\hline 100 & Tortas & 1/2 Troz. / 62g & 1 Troz. / 124g & 3 Troz. / 372g \\
\hline
\end{tabular}

LEYENDA: Reb. = Rebanada; Ud. = Unidad; Tz. = Taza; Cda. = Cucharada; Env. = Envoltorio; Rac. $=$ Ración;

Troz. $=$ Trozo; Cdta. $=$ Cucharadita; Rued. $=$ Rueda; Pza. $=$ Pieza; Filet. $=$ Filete; Beb. $=$ Bebida 
En la Figura 1 se muestra un segmento del atlas diseñado. Cada lámina contempla el tamaño de las porciones en orden ascendente, el gramaje de los alimentos en peso neto o mililitros, así como su medida práctica.

En la Tabla 2, se pueden observar las apreciaciones realizadas al instrumento durante el proceso de valoración por expertos, quienes juzgaron el contenido del instrumento, concluyendo que era adecuado y presentaba claridad en cada uno de los ítems de alimentos considerados.

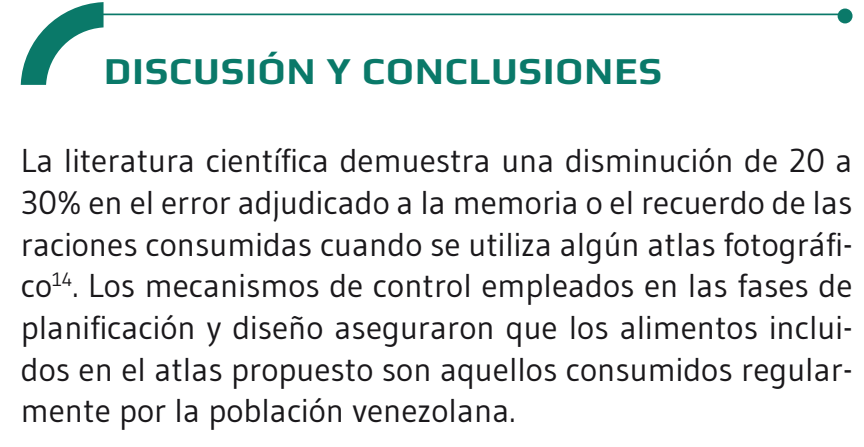

Figura 1. Ejemplos de alimentos incluidos en el atlas fotográfico.

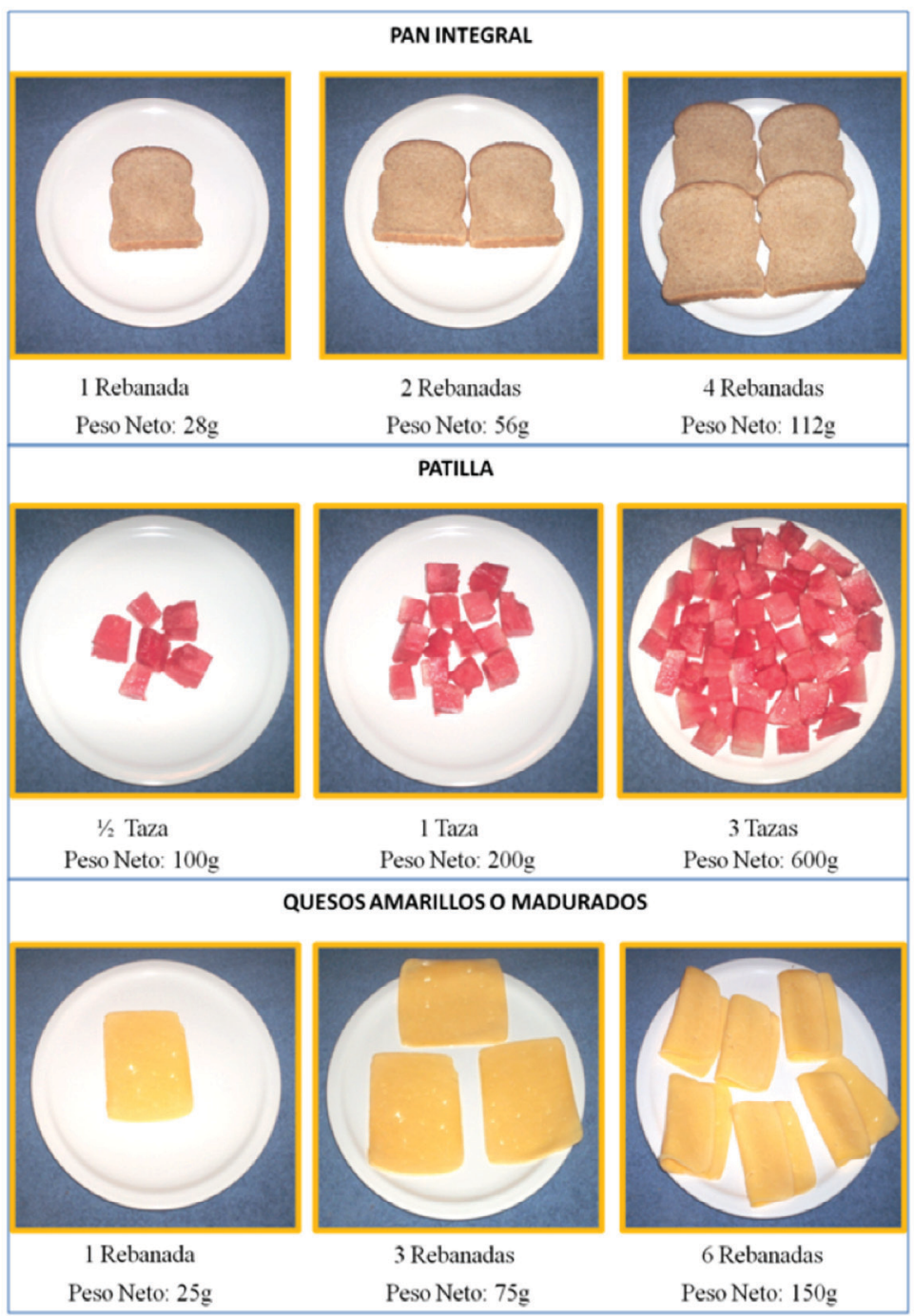

Se muestran las 3 raciones establecidas con medidas prácticas y cantidad en gramos. 
Tabla 2. . Aspectos evaluados por parte de los expertos consultados.

\begin{tabular}{l|l|l|l} 
Aspecto & Experto 1 & Experto 3 \\
\hline $\begin{array}{l}\text { Ajuste de la lista de alimentos } \\
\text { al objetivo del instrumento } \\
\text { propuesto }\end{array}$ & $\begin{array}{l}18 \text { años de experiencia en } \\
\text { Consumo de alimentos }\end{array}$ & $\begin{array}{l}27 \text { años de experiencia en } \\
\text { en Nutrición en Salud } \\
\text { Pública }\end{array}$ & Nutrición Clínica \\
\hline $\begin{array}{l}\text { Relevancia de la información que } \\
\text { aporta el instrumento propuesto }\end{array}$ & Dedianamente de acuerdo acuerdo & De acuerdo & De acuerdo \\
\hline $\begin{array}{l}\text { Adecuación de las fuentes de } \\
\text { información empleadas }\end{array}$ & De acuerdo & De acuerdo & Medianamente de acuerdo \\
\hline $\begin{array}{l}\text { Adecuación del enfoque } \\
\text { metodológico }\end{array}$ & De de acuerdo & De acuerdo \\
\hline $\begin{array}{l}\text { Pertinencia del objetivo } \\
\text { planteado }\end{array}$ & De acuerdo & De acuerdo & De acuerdo
\end{tabular}

La presentación de las raciones de alimentos se hizo en orden ascendente, sin colocar etiquetas de las porciones "pequeña, mediana o grande", acorde a lo recomendado ${ }^{15}$, pues es frecuente la sobre estimación de las pociones pequeñas y la sub estimación de las grandes, cuando se añaden estos rótulos ${ }^{14}$.

Aunque en investigaciones anteriores no se reportaron cambios en la estimación de las raciones si se utilizaba un fondo blanco o negro ${ }^{15}$, en esta experiencia se decidió utilizar una base azul para dar contraste de color a las imágenes y evitar el color blanco similar al del plato. Tueni et al. ${ }^{16}$, también realizaron un atlas con un color de contraste con muy buenos resultados en su validación.

El atlas fotográfico presentado es el producto obtenido en la primera fase del desarrollo de instrumentos. Para su recomendación como herramienta de ayuda en la estimación del tamaño de porciones de alimento consumidas, es necesario validarlo en una muestra de la población objetivo y aplicar procedimientos estadísticos que den garantía de las condiciones técnicas de validez y confiabilidad. Actualmente, este instrumento se encuentra en un proceso de validación y reproducibilidad, como herramienta complementaria de un Cuestionario Semicuantitativo del Consumo de Alimentos, diseñado para la población adulta sana.
La valoración de los expertos apunta a favor de la utilización del instrumento propuesto como herramienta de ayuda en la estimación de las porciones de alimento consumidas. Tal como lo señalan algunas referencias bibliográficas, ya son varios los instrumentos del consumo de alimentos en los cuales se considera importante los comentarios o validación de los expertos ${ }^{17-20}$. Por otro lado, se añade la ventaja de los controles de calidad utilizados durante la planificación y diseño, enfocando los esfuerzos al objetivo, con la revisión sistemática de los materiales de referencia nacional y experiencias similares realizadas en otras partes del mundo. Está muy bien establecido que la vigilancia de los aspectos técnicos y metodológicos durante la planificación contribuye a la optimización de la precisión y validez de los datos obtenidos ${ }^{21}$.

El atlas fotográfico de alimentos desarrollado, según la opinión de los expertos revisores, podría resultar una herramienta útil para ser usada en la evaluación del consumo alimentario en adultos venezolanos, tanto en estudios epidemiológicos como en la práctica clínica. Sin embargo, considerando que todo instrumento debe ser validado en el contexto donde se va a aplicar, queda pendiente la determinación de su validez y confiabilidad, como requisitos técnicos indispensables para su utilización. 


\section{BIBLIOGRAFÍA}

1. López L, Longo E, Carballido M, Di Carlo P. Validación del uso de modelos fotográficos para cuantificar el tamaño de las porciones de alimentos. Rev chil nutr. 2006; 33(3): 480-7.

2. Villalobos D, Maury-Sintjago E, Ríos P, Fernández C, García D, Bravo A. Evaluación del estado nutricional en pacientes pediátricos institucionalizados con VIH/SIDA. Pediatr (Asunción). 2011; 38(1): 31-9.

3. Lares M, Velazco Y, Brito S, Hernández P, Mata C. Evaluación del estado nutricional en la detección de factores de riesgo cardiovascular en una población adulta. Rev latinoam hipertens. 2011; 6(1): 1-7.

4. Gibson RS. Principles of Nutritional Assessment, Second Edition. Oxford: Oxford University Press, 2005.

5. Agencia Española de Seguridad Alimentaria y Nutrición; Demométrica, SL. Atlas Fotográfico para la Encuesta Nacional de Alimentación en la población Infantil y Adolescente (ENALIA). Madrid: Autores; 2013.

6. Navarro A, Cristaldo P, Díaz M, Eynard A. Food photography atlas: its suitability for quantifying food and nutrient consumption in nutritional epidemiological research in Cordoba, Argentina. Rev Fac Cien Med Univ Nac Cordoba. 2000; 57(1): 67-74.

7. Ministerio del Poder Popular para la Alimentación; Instituto Nacional de Nutrición. Tabla de Composición de los Alimentos. Revisión 2012. Colección Seguridad y Soberanía Alimentaria "Edgar Abreu Olivo". Caracas: Fondo Editorial Gente de Maíz; 2012.

8. Instituto Nacional de Nutrición (INN). Hoja de Balance de Alimentos 2009. Caracas: Ministerio del Poder Popular para la Alimentación; 2011.
9. Instituto Nacional de Estadística (INE). [Página web en línea]. Encuesta de Seguimiento al Consumo de Alimentos. Publicaciones segundo semestre 2010 al segundo semestre 2012. [citado en 2014 Oct 10] Disponible en: http://www.ine. gob.ve/

10. Instituto Nacional de Estadística (INE). Encuesta nacional de consumo de alimentos. Boletín informativo INE. 2013; 1: 1-4.

11. Banco Central de Venezuela. [Documento en línea]. IV Encuesta de Presupuestos familiares 2008-2009. Hábitos alimenticios del venezolano 2011. Abril 2011. [citado en 2014 Oct 10] Disponible en: www.bcv.org.ve/epf0809/resultepfiv.ppt

12. Universidad Central de Venezuela, Facultad de Medicina Escuela de Nutrición y Dietética. Tabla de Raciones de Alimentos. Caracas: Autores, 2002.

13. Lazarte C, Encinas M, Alegre C, Granfeldt Y. Validation of digital photographs, as a tool in 24-h recall, for the improvement of dietary assessment among rural populations in developing countries. Nutr J. 2012; 11: 61.

14. Nelson M, Atkinson M, Darbyshire S. Food photography 1: The perception of food portion size from photographs. Br ] Nutr. 1994; 72: 649-63.

15. Nelson M, Haraldsdóttir J. Food photographs: practical guidelines II. Development and use of photographic atlases for assessing food portion size. Public Health Nutr. 1998; 1(4): 231-7.

16. Tueni M, Mounayar A, Birlouez-Aragon I. Development and evaluation of a photographic atlas as a tool for dietary assessment studies in Middle East cultures. Public Health Nutr. 2012; 15(6): 1023-8.

17. Vázquez $M$, Witriw $A$, Editores. Guías de modelos visuales y Tablas de relación Peso/Volumen. Buenos Aires: autores; 1997.

18. de Boer EJ, Slimani N, van't Veer $P$, Boeing $H$, Feinberg $M$, Leclercq $C$, et al. Rationale and methods of the European Food Consumption Validation (EFCOVAL) Project. Eur ] Clin Nutr. 2011; 65(Suppl 1): S1-4.

19. Chaidez V, Kaiser L. Validation of an instrument to assess toddler feeding practices of Latino mothers. Appetite. 2011; 57(1): 229-36.

20. Dickson-Spillmann M, Siegrist M, Keller C. Development and validation of a short, consumer-oriented nutrition knowledge questionnaire. Appetite. 2011; 56(3): 617-20.

21. Alvarez M, Velazco Y. Evaluación del consumo de alimentos en niños y adolescentes. Caracas: Centro de Atención Nutricional Infantil de Antímano; 2010. 